\title{
Age- and gender-specific Prevalence of Reduced Visual Acuity in Children from a Representative Urban District in China from 2002 to 2018
}

\section{Shiting Xiang}

Hunan Children's Hospital https://orcid.org/0000-0002-8191-6541

Sha Zhao

Hunan Children's Hospital

Xun Li

Hunan Children's Hospital

Liping Li

Hunan Children's Hospital

Lihua Xie

Hunan Children's Hospital

\section{Rutong Kang}

Hunan Children's Hospital

Yan Zhong ( $\nabla$ yz_hnch@163.com )

\section{Research article}

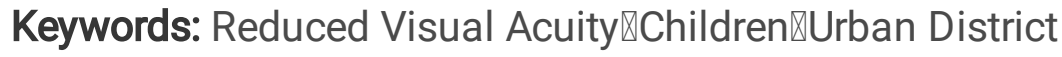

Posted Date: September 5th, 2019

DOI: https://doi.org/10.21203/rs.2.13909/v1

License: (c) (i) This work is licensed under a Creative Commons Attribution 4.0 International License. Read Full License 


\section{Abstract}

Background To investigate age- and gender-specific trends in the prevalence of reduced visual acuity (VA), a proxy measure for myopia, in a representative urban district in China. And to identify the susceptible age for the emerging of reduced VA epidemic. Methods Data were extracted from the dataset of 2002 and 2018 Annual Survey on Students' Constitution and Health from Yuhua District, Changsha City, China. Children aged 6 to 15 years were included. The visual acuity was measured by a LogMAR tumbling $E$ chart. The prevalence of reduced VA was calculated by age and gender. Cochran-Armitage test was used for detecting trends. Results The final VA analysis included 26217 children in 2002囚and 45510 children in 2018. The overall prevalence of reduced VA increased from $28.3 \%$ in 2002 to $46.5 \%$ in $2018(\mathrm{P}<0.001)$. The prevalence of reduced VA started to increase markedly from the age of 14 years in 2002, and in 2018 it started to increase at the age of 9 years. The prevalence of severely reduced VA increased in all age groups from 2002 to 2018 and increased with age (all $P<0.001$ ). Between age 6 to 15 years, the proportion of normal VA decreased from $61.4 \%$ to $31.9 \%$ in 2018 . Conclusions Both the prevalence of reduced VA and severely reduced VA in children aged 6-15 years old increased significantly from 2002 to 2018 in Yuhua District. The remarkable epidemic of reduced VA started 5 years earlier in 2018 than in 2002. Evidence from the present study suggests interventions should be launched before the age of 9 years.

\section{Background}

Myopia is the most common eye disease affecting the eyesight of children and adolescents [1]. It not only damages physical and mental health, learning and working ability, but also increases the risk of eye complications, including retinopathy, glaucoma, retinal detachment, and blindness [2, 3]. Many epidemiological studies have confirmed that a high educational level of children and parents, female, less outdoor activity, and near work are associated with myopia [4-6].

In the east and southeast Asia, where $80-90 \%$ of the adolescents are affected by myopia, the myopia epidemic has become a public health threat $[7,8]$. Therefore, it is one of the main priorities of the five vision 2020 initiatives launched by WHO[9]. China, with its rapid economic development and transitions in behavior and lifestyle, is one of the countries with the highest rates of childhood myopia in the world [10]. According to surveys, the prevalence of myopia among adolescents is about $95.5 \%$ in Shanghai and $74.2 \%$ in Beijing $[11,12]$. The Chinese government pays more attention than ever before to the eyesight health of children and adolescents.

Identifying at what age the prevalence of myopia starts to increase is important for the development of interventions. Also, comparing the age-specific prevalence of myopia overtime can help pinpoint the ages in childhood when the eyesight is most sensitive to change. Using visual examination data from the dataset of 2002 and 2018 Annual Survey on Students' Constitution and Health from Yuhua District, Changsha City, China, the purpose of this study was to investigate age- and gender-specific trends in the prevalence of reduced visual acuity (VA), a proxy measure for myopia, in a representative urban district in 
China. And therefore provide references for the development of interventions to stop the increasing trend of myopia in China and in other countries that encountered similar challenges.

\section{Methods}

\section{Study subjects}

Changsha, the capital of the Hunan province of central China, has more than 3.5 million residents living in six urban districts. It has well developed elementary and secondary education system, all school-age children will join the nine-year compulsory education in elementary school (6 years) and secondary school (3 years). We performed the analysis using data extracted from the dataset of 2002 and 2018 Annual Survey on Students' Constitution and Health from Yuhua District, Changsha City, China. The random cluster sampling method was used to select elementary and secondary schools from Yuhua District. All students from the selected schools underwent visual examinations according to the manual of the National Student Constitution and Health Survey (Chinese version). Children aged less than 6 years or older than 15 years from the dataset were excluded from the analysis. We collected general information including name, sex, age, and the name of their school of all participants.

\section{VA determinations}

The visual acuity was measured by a retro-illuminated LogMAR chart with tumbling-E optotypes (Precision Vision, La Salle, IL, USA). The specific measurement method was as follows: (1) The fourth line from the bottom (6/6) should be equal to the visual altitude of the test eye. (2) Both eyes were examined separately, first the right eye, then the left eye. (3) Measurements started at a distance of $5 \mathrm{~m}$, starting from the fourth line from the bottom (6/6), using a staircase protocol, four of the five optotypes identified considered as correct. (4) The VA equals the lowest line reading successfully. (5) If the top line was not read, the child would move forward until he could read. The protocol of VA examinations was the same in both surveys. Presenting and best-corrected VA were not measured.

Normal VA was defined as equal to or greater than $6 / 6$. Mildly reduced VA was defined as greater than $6 / 9$ and less than $6 / 6$. Moderately reduced VA was defined as greater than $6 / 18$, equal to or less than $6 / 9$, and severely reduced VA was defined as equal to or less than 6/18.

\section{Statistical analysis}

Prevalence of reduced VA and severely reduced VA were calculated by age and gender. Chi-square test was used to compare the differences between groups. The Cochran-Armitage test was used to examine the age trends for the prevalence of severely reduced VA. All statistical analyses were performed using SPSS version 22.0 (IBM, New York, USA). The figures were plotted using Microsoft Excel 2007. All of the statistical tests were two-sided. All $P$ values were 2 -sided and $P<0.05$ was considered significant for all tests. 


\section{Results}

A total of 26644 children in 2002 and 45696 in 2018 were measured. 427 children in 2002 and 186 children in 2018 were excluded from the analysis because of incomplete information. The final analysis included 26217 children in 2002, and 45510 children in 2018. There were no significant differences in age and sex between the two surveys ( $P>0.05)$.

Table 1 shows that the overall prevalence of reduced VA increased from $28.3 \%(95 \% \mathrm{Cl}, 27.8 \%-28.9 \%)$ in 2002 to $46.5 \%(95 \% \mathrm{Cl}, 46.0 \%-46.9 \%)$ in $2018(P<0.001)$.

Table 2 shows the prevalence of reduced VA stratified by age and sex.

Reduced VA was more prevalent across age groups in 2018 than in 2002, except for $6 \sim$ to $8 \sim$ year age group $(P<0.001)$. The prevalence of reduced VA in boys increased from $24.8 \%$ in 2002 to $43.2 \%$ in 2018 , and from $32.3 \%$ to $50.2 \%$ for girls $(P<0.001)$.

Fig1 shows reduced VA prevalence of girls was higher than boys in all age groups. In 2018, the prevalence of reduced VA started to increase markedly from the age of 9 years, while in 2002, the prevalence started to increase markedly from the age of 14 years.

Table 3 shows the prevalence of severely reduced VA increased in all age groups from 2002 to 2018 (all $P$ $<0.001$ ). Between age 6 to 15 years, the prevalence of severely reduced VA ranged from $0.8 \%$ to $14.9 \%$ in 2002 , while in 2018 , the prevalence increased from $1.5 \%$ to $38.6 \%$ (all $P<0.001$ ).

In 2002, normal VA accounted for the majority of all age groups. In 2018, the proportion of normal VA decreased from $61.4 \%$ to $31.9 \%$ respectively (Fig 2 and Fig 3 ).

\section{Discussion}

In this population-based study on school children aged 6-15 years old in Changsha, we found that there has been a significant increase in the overall prevalence of reduced unaided VA from 2002 to 2018. In $2002,71.7 \%$ of children had normal VA, while only $53.5 \%$ had normal VA in 2018 . Similar trends are seen in other studies conducted among school children in China [13-16]. Among Chinese children in Guangzhou, by the end of schooling, less than 15\% of children had normal VA in 2007 compared with around $30 \%$ in 1988[13]. Sun reported the overall prevalence of reduced VA of Chinese Students increased from $28.6 \%$ to $56.8 \%$ from 1985 to 2010 in mainland China [14].

Unaided VA was a justified measure of myopia because of its satisfactory sensitivity and specificity in myopia screening in previous studies $[13,14]$. Therefore, although cycloplegic refraction (the gold standard for myopia assessment) was not measured due to the large sample size, the observed trend of the increase was largely due to the increased prevalence of myopia. In China, visual impairment is usually caused by refractive error, and myopia is the most common form of refractive error in school-age children[17]. 
Compared to 2002, our results indicated that both the prevalence of reduced VA and severely reduced VA increased significantly across almost all age and gender groups of children in Changsha in 2018. The results showed that the age-specific prevalence of reduced VA had an obvious trend that it increases with age. We also observed that girls had a higher prevalence of reduced VA than boys, which is consistent with findings from other studies [18-21]. The previous study showed that women are 3.9 times more likely than men to suffer from refractive errors [19]. In traditional Chinese culture, girls are expected to be quiet. They spend more time indoors doing near work while boys spend more time outdoors which may prevent the development of myopia [22].

Our results also showed that the proportion of severely reduced VA in 2018 increased more rapidly as children aged, which was consistent with previous research results[23,24]. In China, the intensity of studying increases with age. Therefore, they have to spend more time doing homework every day and less time playing outdoors. Studies have shown that the intensity of studying is the main environmental and behavioral factor of myopia[21].

To our knowledge, this is the first study focusing on the vision of elementary and secondary school students in central China. However, there were some limitations. First, because of the large sample size, we did not perform refractive examination. We used unaided VA as an alternative measure of myopia because of its satisfactory sensitivity and specificity in myopia screening[13,14]. Second, rather than using a national representative sample, we used a sample from a representative urban district from China, therefore our result may not able to reflect the trends of reduced VA in rural China. Also, the prevalence rate in Yuhua District could be different from the national prevalence rate in China, because there are regional differences. However, the main purpose of our study was to identify the changes in agespecific trends, and our findings should be able to reflect the trends in urban China. It could also be used as a reference for other Asia countries that facing an epidemic of myopia.

\section{Conclusion}

Both the prevalence of reduced VA and severely reduced VA in children aged 6-15 years old increased significantly from 2002 to 2018 in Yuhua District. The remarkable epidemic of reduced VA started 5 years earlier in 2018 than in 2002. Evidence from the present study suggests interventions should be launched before the age of 9 years.

\section{Abbreviations}

VA :Reduced visual acuity

WHO: World health organization

\section{Declarations}

Ethics approval and consent to participate 
This study was approved by the Ethics Committee of the Hunan Children's Hospital. Informed written consent was obtained from a legal guardian of each child involved in our study.

\section{Consent to publish}

All authors approved the final manuscript for submission and gave consent for publication.

\section{Availability of data and materials}

The datasets used and analysed during the current study are available from the corresponding author on reasonable request.

\section{Competing interests}

The authors declare that they have no competing interests.

\section{Funding}

No funding was given to the study.

\section{Authors' contributions}

STX and SZ contributed equally to designing this article, performing the statistical analyses, and drafting the manuscript. XL provided critical revision of the manuscript. YZ and LPL participated in the design of the study. LHX and RTK helped completed the acquisition of data. All authors read and gave final approval of the version to be published.

\section{Acknowledgements}

The authors thank the subjects in the study and appreciate all of the support of the data collectors.

\section{References}

1). Resnikoff S, Pascolini D, Mariotti SP, Pokharel GP. Global magnitude of visual impairment caused by uncorrected refractive errors in 2004. Bull World Health Organ. 2008;86(1):63-70. doi: $10.2471 /$ blt.07.041210

2). Saw SM, Gazzard G, Shih-Yen EC, Chua WH. Myopia and associated pathological complications. Ophthalmic Physiol Opt. 2005;25(5):381-91. doi: 10.1111/j.1475-1313.2005.00298.x

3). Morgan IG, Ohno-Matsui K, Saw SM. Myopia. LANCET. 2012;379(9827):1739-48.doi:

10.1016/S0140-6736(12)60272-4

4) Wu LJ, Wang YX, You QS, Duan JL, Luo YX, Liu LJ, et al. Risk Factors of Myopic Shift among Primary School Children in Beijing, China: A Prospective Study. INT J MED SCl. 2015;12(8):633-8. doi: 
5). Saw SM, Chan YH, Wong WL, Shankar A, Sandar M, Aung T, et al. Prevalence and risk factors for refractive errors in the Singapore Malay Eye Survey. Ophthalmology. 2008 Oct;115(10):1713-9. doi:10.1016/j.ophtha.2008.03.016

6). Rose KA, Morgan IG, Smith W, Burlutsky G, Mitchell P, Saw SM. Myopia, lifestyle, and schooling in students of Chinese ethnicity in Singapore and Sydney. Arch Ophthalmol. 2008; 126:527-530. doi: 10.1001/archopht.126.4.527 PMID: 18413523

7). Bourne RRA, Flaxman SR, Braithwaite T, Cicinelli MV, Das A, Jonas JB, et al. Magnitude, temporal trends, and projections of the global prevalence of blindness and distance and near vision impairment: a systematic review and meta-analysis. The Lancet Global Health. 2017;5(9):e888-97. doi: 10.1016/S2214109X(17)30293-0

8). Morgan IG, French AN, Ashby RS, Guo X, Ding X, He M, et al. The epidemics of myopia: Aetiology and prevention. PROG RETIN EYE RES. 2018;62:134-49. doi: 10.1016/j.preteyeres.2017.09.004

9). He M, Abdou A, Naidoo KS, Sapkota YD, Thulasiraj RD, Varma R, et al. Prevalence and correction of near vision impairment at seven sites in China, India, Nepal, Niger, South Africa, and the United States. AM J OPHTHALMOL. 2012;154(1):107-16. doi: 10.1016/j.ajo.2012.01.026

10). Dolgin E. The myopia boom. NATURE. 2015;519(7543):276-8. doi: 10.1038/519276a

11). Sun J, Zhou J, Zhao P, Lian J, Zhu H, Zhou Y, et al. High prevalence of myopia and high myopia in 5060 Chinese university students in Shanghai. Invest Ophthalmol Vis Sci. 2012;53(12):7504-9. doi: 10.1167/iovs.11-8343

12). You QS, Wu LJ, Duan JL, Luo YX, Liu LJ, Li X, et al. Prevalence of myopia in school children in greater Beijing: the Beijing Childhood Eye Study. ACTA OPHTHALMOL. 2014;92(5):e398-406.

13) .Xiang F, He M, Zeng Y, Mai J, Rose KA, Morgan IG. Increases in the prevalence of reduced visual acuity and myopia in Chinese children in Guangzhou over the past 20 years. Eye (Lond). 2013;27(12):1353-8. doi: 10.1038/eye.2013.194

14). Sun H, Li A, Xu Y, Pan C. Secular Trends of Reduced Visual Acuity From 1985 to 2010 and Disease Burden Projection for 2020 and 2030 Among Primary and Secondary School Students in China. JAMA OPHTHALMOL. 2015;133(3):262. doi: 10.1001/jamaophthalmol.2014.4899

15). Jan C, Xu R, Luo D, Xiong X, Song Y, Ma J, et al. Association of Visual Impairment With Economic Development Among Chinese Schoolchildren. JAMA PEDIATR. 2019:e190914. doi:

10.1001/jamapediatrics.2019.0914 
16). Li Y, Liu J, Qi P. The increasing prevalence of myopia in junior high school students in the Haidian District of Beijing, China: a 10-year population-based survey. BMC OPHTHALMOL. 2017;17(1). doi: 10.1186/s12886-017-0483-6

17). He $M$, Huang $W$, Zheng $Y$, Huang $L$, Ellwein LB. Refractive error and visual impairment in school children in rural southern China. Ophthalmology. 2007;114(2):374-382.

18). Huang L, Kawasaki H, Liu Y, Wang Z. The prevalence of myopia and the factors associated with it among university students in Nanjing: A cross-sectional study. Medicine (Baltimore). 2019;98(10):e14777. doi: 10.1097/MD.0000000000014777

19). Sewunet SA, Aredo KK, Gedefew M. Uncorrected refractive error and associated factors among primary school children in Debre Markos District, Northwest Ethiopia. BMC OPHTHALMOL. 2014;14:95. doi: 10.1186/1471-2415-14-95

20). Saw SM, Chan YH, Wong WL, Shankar A, Sandar M, Aung T, et al. Prevalence and risk factors for refractive errors in the Singapore Malay Eye Survey. OPHTHALMOLOGY. 2008;115(10):1713-9. doi: 10.1016/j.ophtha.2008.03.016

21). You QS, Wu LJ, Duan JL, Luo YX, Liu LJ, Li X, et al. Factors associated with myopia in school children in China: the Beijing childhood eye study. PLOS ONE. 2012;7(12):e52668. doi: 10.1371/journal.pone.0052668

22) .He M, Xiang F, Zeng Y, Mai J, Chen Q, Zhang J, et al. Effect of Time Spent Outdoors at School on the Development of Myopia Among Children in China. JAMA. 2015;314(11):1142. doi:

10.1001/jama.2015.10803

23). Guo Y, Duan JL, Liu LJ, Sun Y, Tang P, Lv YY, et al. High myopia in Greater Beijing School Children in 2016. PLOS ONE. 2017;12(11):e187396. doi: 10.1371/journal.pone.0187396

24). Hashim SE, Tan HK, Wan-Hazabbah WH, Ibrahim M. Prevalence of refractive error in malay primary school children in suburban area of Kota Bharu, Kelantan, Malaysia. Ann Acad Med Singapore. 2008;37(11):940-6.

\section{Tables}

Table 1 General characteristics of the study participants in 2002 and 2018 


\begin{tabular}{|c|c|c|c|c|c|}
\hline \multirow[t]{2}{*}{ Category } & \multicolumn{2}{|r|}{2002} & \multicolumn{2}{|r|}{2018} & \multirow[t]{2}{*}{$\bar{P}$} \\
\hline & $\mathrm{n}$ & $\%(95 \% \mathrm{CI})$ & $\mathrm{n}$ & $\%(95 \% \mathrm{CI})$ & \\
\hline $\begin{array}{c}\text { Age(y) } \\
6 \sim 11 \\
12 \sim 15\end{array}$ & $\begin{array}{l}17583 \\
9061\end{array}$ & $\begin{array}{l}66.0(65.4,66.6) \\
34.0(33.4,34.6)\end{array}$ & $\begin{array}{l}29968 \\
15728\end{array}$ & $\begin{array}{l}65.6(65.1,66.0) \\
34.4(34.0,34.9)\end{array}$ & 0.261 \\
\hline $\begin{array}{l}\text { Sex } \\
\text { Boys }\end{array}$ & 14127 & 3.6) & 24409 & $53.4(53 .($ & 0.305 \\
\hline Girls & 12517 & $47.0(46.4,47.6)$ & 21287 & $46.6(46.1,47.0)$ & 0.00 \\
\hline Visual acuity & & & & & \\
\hline Normal & 18786 & $71.7(71.1,72.2)$ & 24357 & $53.5(53$. & $<0.001$ \\
\hline $\begin{array}{l}\text { Reduced }^{\mathrm{a}} \\
\text { Reduced Visual acuity }\end{array}$ & 7431 & 28.3(27.8,28.9) & 21153 & 46. & \\
\hline Mildly reduced ${ }^{b}$ & 3557 & $47.9(46.7,49.0)$ & 5704 & $27.0(26.4,27.6)$ & $<0.001$ \\
\hline Moderately reduced $^{C}$ & 2762 & $37.2(36.1,38.3)$ & 7978 & $37.7(37.1,38.4)$ & \\
\hline Severely reduced ${ }^{\mathrm{d}}$ & 1112 & $15.0(14.2,15.8)$ & 7471 & $35.3(34.7,36.0)$ & \\
\hline
\end{tabular}
a. Reduced visual acuity was defined as worse than 6/6.
b. Mildly reduced VA was defined as greater than $6 / 9$ and less than $6 / 6$.
c. Moderately reduced VA was defined as greater than $6 / 18$, equal to or less than $6 / 9$
d. severely reduced VA was defined as equal to or less than $6 / 18$

Table 2 Prevalence of reduced visual acuity stratified by sex and age among students aged $6 \sim 15$ years

\begin{tabular}{|c|c|c|c|c|c|c|c|}
\hline \multirow[t]{2}{*}{ Category } & \multicolumn{3}{|c|}{2002} & \multicolumn{3}{|c|}{2018} & \multirow[b]{2}{*}{$P$} \\
\hline & $\mathrm{n}$ & Total & $\%(95 \% \mathrm{CI})$ & $\mathrm{n}$ & Total & $\%(95 \%$ CI $)$ & \\
\hline $\operatorname{Age}_{6}(\mathrm{y})$ & 14 & 914 & $45.4(4$ & 4845 & 1872 & $0)$ & $<0001$ \\
\hline 7 & 2019 & 1038 & & 5714 & & , 33.6$)$ & 0.003 \\
\hline 8 & 3091 & 880 & $28.5(26.9,30.1)$ & 5379 & 1648 & $30.6(29.4,31.9)$ & 0.036 \\
\hline 9 & 3133 & 734 & $23.4(21.9,24.9)$ & 4773 & 1728 & $36.2(34.8,37.6)$ & $<0.001$ \\
\hline 10 & 2957 & 688 & $.7,24.8)$ & 4648 & 1904 & $41.0(39.5,42.4)$ & $<0.001$ \\
\hline 11 & 3185 & 694 & 21.8 & 4470 & 2155 & ,49.7) & $<0.001$ \\
\hline 12 & 3197 & 716 & $.0,23.8)$ & 5096 & 2887 & $56.7(55.3,58.0)$ & $<0.001$ \\
\hline 13 & 2378 & 559 & $23.5(21.8,25.2)$ & 5113 & 3312 & $3.5,66.1)$ & $<0.001$ \\
\hline 14 & 186 & 609 & $.6,34.8)$ & 4680 & 3259 & $8.3,71.0)$ & $<0.001$ \\
\hline 15 & 1481 & 599 & $(7.9,42.9)$ & 792 & 539 & $64.8,71.3)$ & $<0.001$ \\
\hline \multicolumn{8}{|l|}{ Sex } \\
\hline Boys & 878 & 3447 & $24.8(24$ & 24293 & 104 & $43.2(4$ & $<0.001$ \\
\hline Girls & 12339 & 3984 & $32.3(31.5,33.2)$ & 21217 & 10657 & $50.2(49.6,50.9)$ & $<0.001$ \\
\hline
\end{tabular}

Table 3 Prevalence of severely reduced visual acuity stratified by sex and age among students aged 6 15 years 


\begin{tabular}{|c|c|c|c|c|c|c|c|}
\hline \multirow[t]{2}{*}{ Category } & \multicolumn{3}{|c|}{2002} & \multicolumn{3}{|c|}{2018} & \multirow[b]{2}{*}{$\bar{P}$} \\
\hline & $\mathrm{n}$ & Total & $\%(95 \% \mathrm{CI})$ & $\mathrm{n}$ & Total & $\%(95 \%$ CI $)$ & \\
\hline Age(y) & & & & & & & \\
\hline 6 & 2014 & 17 & $0.8(0.4,1.2)$ & 4845 & 74 & $1.5(1.2,1.9)$ & $<0.001$ \\
\hline 7 & 2919 & 32 & $1.1(0.7,1.5)$ & 5714 & 129 & $2.3(1.9,2.6)$ & $<0.001$ \\
\hline 8 & 3091 & 40 & $1.3(0.9,1.7)$ & 5379 & 208 & $3.9(3.4,4.4)$ & $<0.001$ \\
\hline 9 & 3133 & 71 & $2.3(1.7,2.8)$ & 4773 & 378 & $7.9(7.2,8.7)$ & $<0.001$ \\
\hline 10 & 2957 & 95 & $3.2(2.6,3.8)$ & 4648 & 522 & $11.2(10.3,12.1)$ & $<0.001$ \\
\hline 11 & 3185 & 122 & $3.8(3.2,4.5)$ & 4470 & 833 & $18.6(17.5,19.8)$ & $<0.001$ \\
\hline 12 & 3197 & 176 & $5.5(4.7,6.3)$ & 5096 & 1365 & $26.8(25.6,28.0)$ & $<0.001$ \\
\hline 13 & 2378 & 162 & $6.8(5.8,7.8)$ & 5113 & 1773 & $34.7(33.4,36.0)$ & $<0.001$ \\
\hline 14 & 1862 & 176 & $9.5(8.1,10.8)$ & 4680 & 1883 & $40.2(38.8,41.6)$ & $<0.001$ \\
\hline 15 & 1481 & 221 & $14.9(13.1,16.7)$ & 792 & 306 & $38.6(35.2,42.0)$ & $<0.001$ \\
\hline \multicolumn{8}{|l|}{ Sex } \\
\hline Boys & 13878 & 470 & $3.4(3.1,3.7)$ & 24293 & 3505 & $14.4(14.0,14.9)$ & $<0.001$ \\
\hline Girls & 12339 & 642 & $5.2(4.8,5.6)$ & 21217 & 3966 & $18.7(18.2,19.2)$ & $<0.001$ \\
\hline
\end{tabular}

\section{Figures}

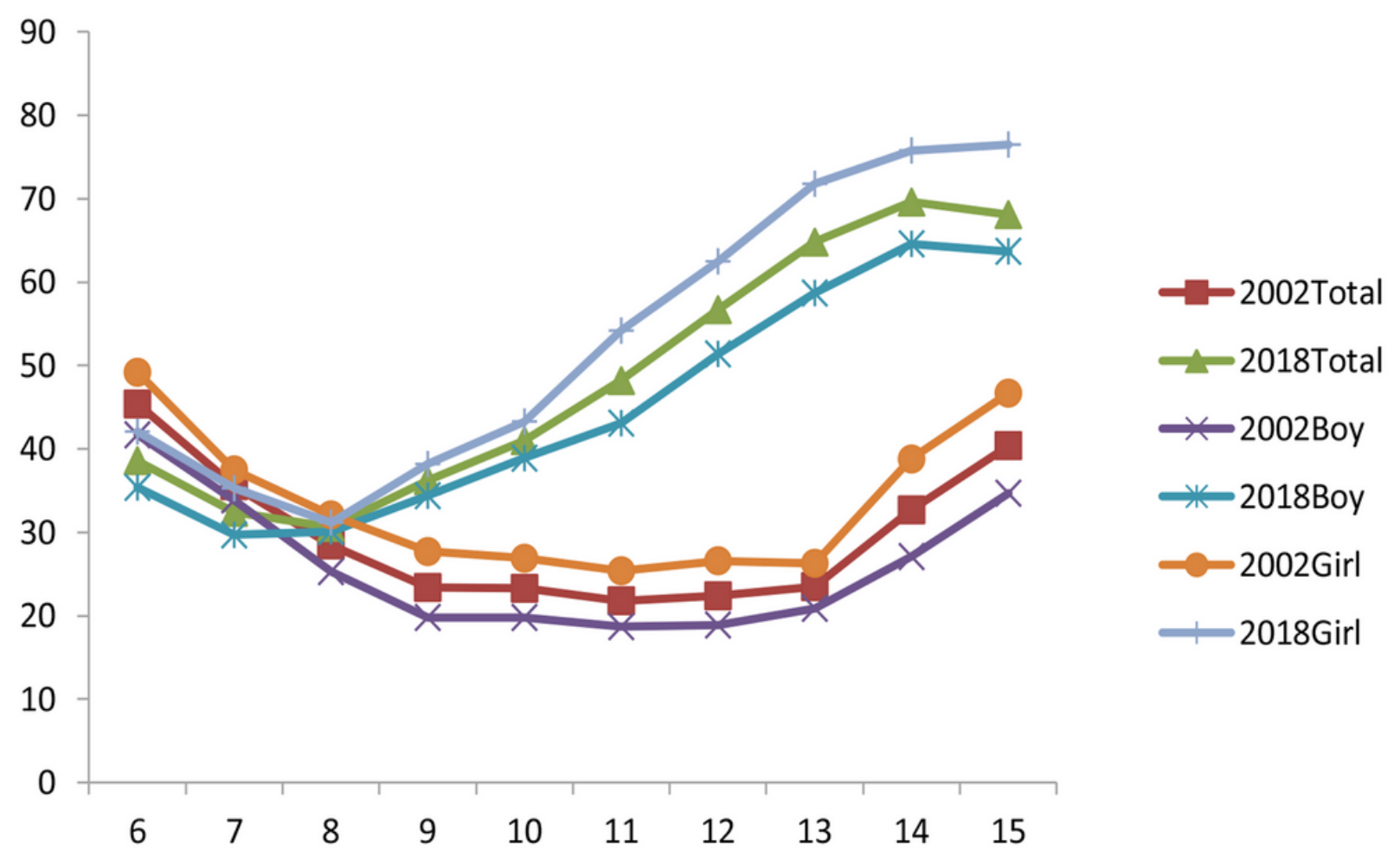

\section{Figure 1}

Prevalence of Reduced Visual Acuity in China by Sex Group in 2002 and 2018 


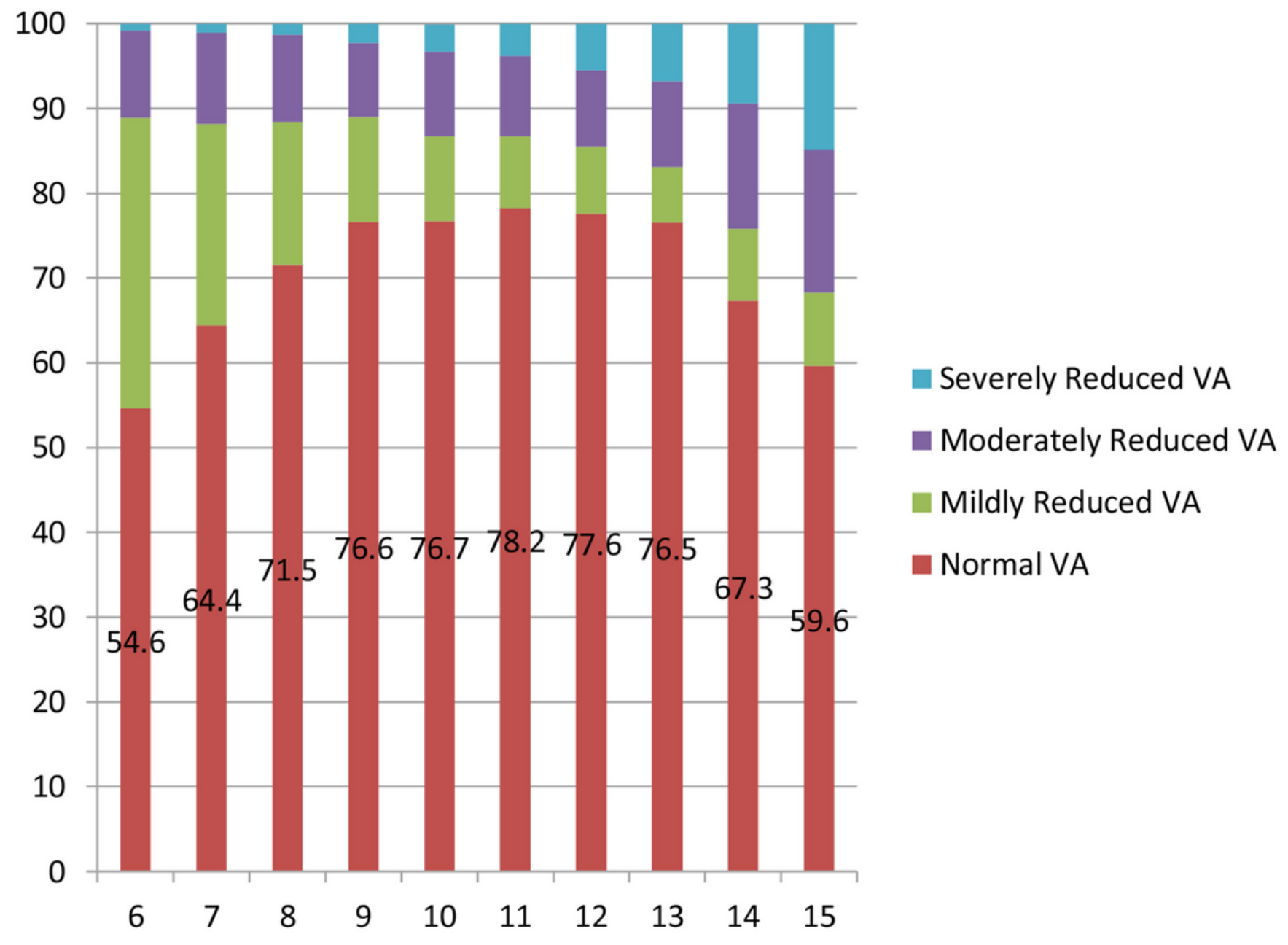

Figure 2

Percentages of Students With Normal, Mildly, Moderately, and Severely Reduced Visual Acuity in 2002 


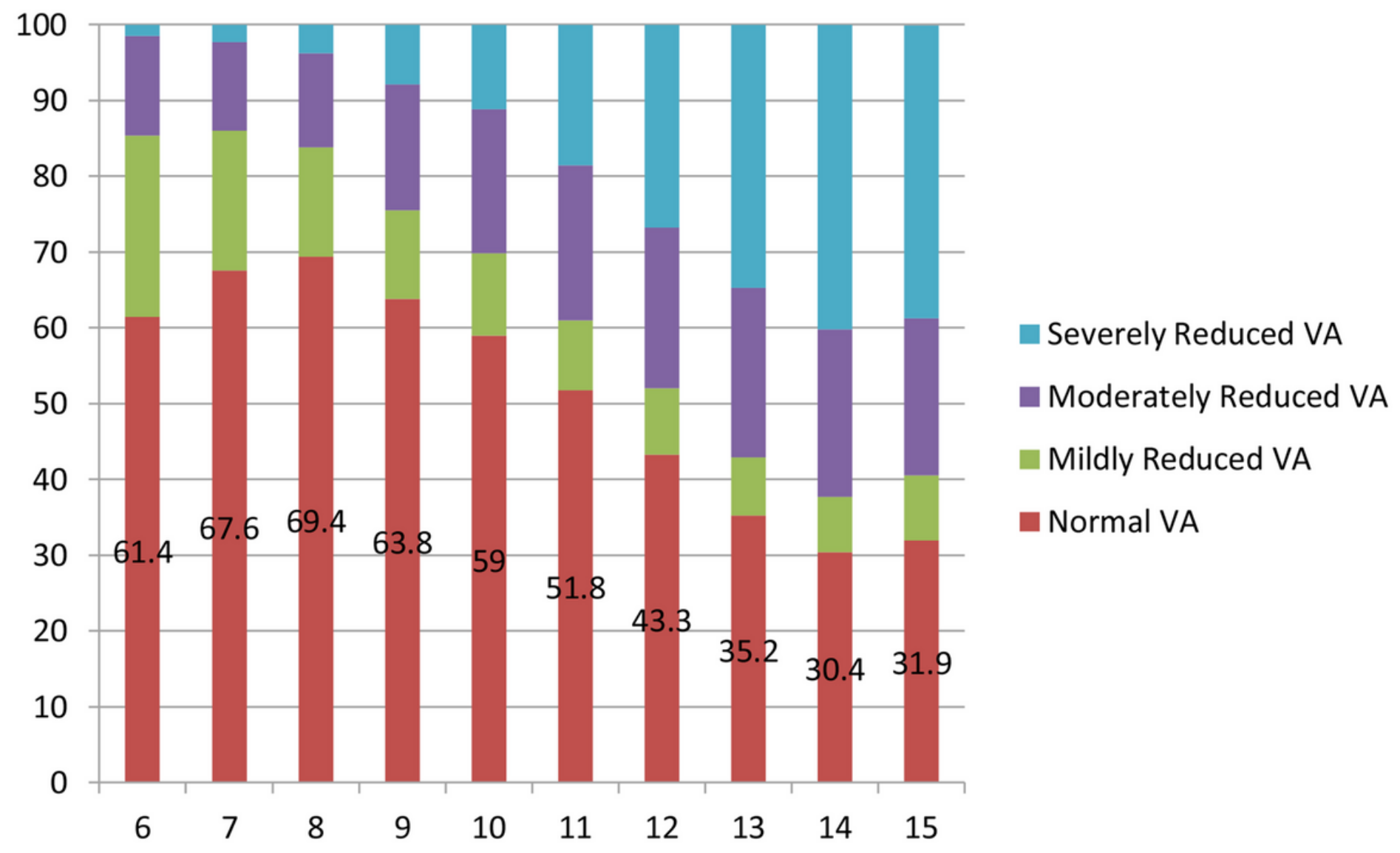

Figure 3

Percentages of Students With Normal, Mildly, Moderately, and Severely Reduced Visual Acuity in 2018 